\title{
Liderazgo en instituciones de educación superior: Un análisis a través de Bibliometrix $\mathbf{R}$
}

\author{
Leadership in higher education institutions: An analysis through Bibliometrix $R$
}

\author{
Liliana Pedraja-Rejas ${ }^{1 *} \quad$ Emilio Rodríguez-Ponce ${ }^{2}$ \\ Andrés Bernasconi $^{3} \quad$ Camila Muñoz-Fritis ${ }^{4}$
}

Recibido 10 de Diciembre de 2020, aceptado 21 de Enero de 2021

Received: December 10, 2020 Accepted: January 21, 2021

\begin{abstract}
RESUMEN
El propósito de este artículo es identificar las principales líneas de investigación del liderazgo en las instituciones de educación superior que han emergido en el período 2019-julio 2020. Para esto se realizó un análisis bibliométrico de las bases de datos Web of Science, Scopus y SciELO bajo los términos de liderazgo, e instituciones de educación superior, universidades e institutos profesionales. Se utilizó la herramienta de Bibliometrix R para describir las tendencias en el campo. Los hallazgos sugieren cuatro áreas principales de estudio asociadas al liderazgo, las cuales son 1) los efectos (directos o indirectos) que tienen los estilos de liderazgo en distintos aspectos de interés, 2) los programas de aprendizaje y desarrollo implementados en las instituciones de educación terciaria para incentivar el liderazgo en los estudiantes y docentes, 3 ) el liderazgo de las mujeres en la academia, y 4) las actitudes, competencias, conocimientos y características que deben presentar los líderes para ser efectivos en el ámbito académico.
\end{abstract}

Palabras clave: Liderazgo, instituciones de educación superior, análisis bibliométrico, bibliometrix.

\begin{abstract}
The aim of this article is to identify the main research lines of leadership in higher education institutions that have emerged in the period 2019-July 2020. For this, a bibliometric analysis of the Web of Science, Scopus, and SciELO databases was carried out under the terms of leadership, and institutions of higher education, universities, and community colleges. Similarly, the Bibliometrix R-tool was used to describe trends in the field. The findings suggest four main areas of study associated with leadership, which are 1) the effects (direct or indirect) that leadership styles have on different aspects of interest, 2) the learning and development programs implemented in tertiary educational institutions to encourage leadership in students and teachers, 3) the women leadership in academia, and 4) the attitudes, competencies, knowledge, and characteristics that leaders must present to be effective in the academic field.
\end{abstract}

Keywords: Leadership, higher education institutions, bibliometric analysis, bibliometrix.

\section{INTRODUCCIÓN}

Las instituciones de educación superior en su misión de crear y transmitir conocimiento avanzado han contribuido significativamente al desarrollo de los recursos humanos de un país, y la inversión en el sector ha desempeñado un papel vital en el crecimiento económico de las naciones [1], es

\footnotetext{
1 Universidad de Tarapacá. Departamento de Ingeniería Industrial y Sistemas. Arica, Chile. E-mail: 1pedraja@uta.cl

2 Universidad de Tarapacá. Instituto de Alta Investigación. Arica, Chile. E-mail: erodriguez@uta.cl

3 Pontificia Universidad Católica de Chile. Facultad de Educación. Santiago, Chile. E-mail: abernasconi@uc.cl

4 Universidad de Tarapacá. Departamento de Ingeniería Industrial y Sistemas. Arica, Chile.

E-mail: camila.munoz.fritis@gmail.com

* Autor de correspondencia: lpedraja@uta.cl
} 
por esto que buscar factores que puedan afectar el cumplimiento de estos objetivos ha tomado gran relevancia en el último tiempo [2]. En este contexto, varios son los autores que han identificado al liderazgo como un factor crítico, ya que el ejercicio efectivo de este puede contribuir al cumplimiento de las metas propuestas y traer beneficios en este tipo de instituciones [3].

En definitiva, el liderazgo es definido como una habilidad práctica que permite el logro de objetivos con una mirada de dirección individual, en equipo u organizacional [4], lo cual en el ámbito académico ocupa un lugar destacado en la agenda de muchas universidades y, por lo tanto, es un tema de importancia para los líderes, administradores, y todos aquellos involucrados en transformar la enseñanza y apoyar a docentes en aspectos pedagógicos [5].

En consecuencia, el liderazgo se ha transformado en un tópico de gran interés empírico [5], y es en este contexto donde diversos investigadores han estudiado este tema desde diferentes perspectivas [2]. Sin embargo, Pedraja-Rejas et al. [6] plantean que el foco central de las investigaciones ha sido encontrar modos de mejora en la gestión institucional. En este sentido, en la literatura existente se pueden encontrar estudios que relacionan el liderazgo con los niveles de satisfacción laboral, manejo de conflictos, y en el logro de la flexibilidad, calidad, efectividad y crecimiento organizacional [3], entre otros.

A continuación, se explora desde un análisis bibliométrico realizado a partir de las bases de datos Web of Science (WoS), Scopus y SciELO, el término de liderazgo en la educación superior con el objeto de poder inferir, desde el análisis de contenido, las principales líneas de investigación que han emergido sobre esta temática en el período 2019-julio 2020.

\section{HACIA LA DEFINICIÓN DE LIDERAZGO EN LAS INSTITUCIONES DE EDUCACIÓN SUPERIOR}

Actualmente, el liderazgo se considera uno de los pilares claves para el logro de la calidad en la educación superior [7], ya que los líderes tienen la capacidad para aclarar roles y responsabilidades, garantizar la asignación adecuada de recursos, crear asociaciones y optimizar la gestión de personas y procesos [8]. En este sentido, Butler [9] sostiene que los líderes académicos deben poseer habilidades técnicas, sociales, emocionales y de gestión, ya que estos están en el centro del logro de la visión, misión y objetivos estratégicos de este tipo de instituciones [2].

Asimismo, en este contexto coexisten distintos niveles de liderazgo los cuales poseen particulares habilidades, como lo son los líderes principales de estas instituciones, líderes de rango medio y los líderes académicos. A continuación, se describen cada uno de ellos.

En el primer grupo se plantea que estos líderes deben orquestar la creación de planes estratégicos $\mathrm{y}$ asignar los recursos acorde a las prioridades que se establezcan en la institución [5], gestionar las demandas y expectativas de las partes interesadas como la academia, la administración, la profesión, los estudiantes, los actores internacionales y gubernamentales [10] y promover una cultura adaptativa que abrace y equilibre la tensión que emerge entre la innovación y operación del día a día [11].

En el segundo grupo se encuentran los líderes medios. Huang et al. [12] plantean que estos cada vez cumplen un papel más importante debido a su proximidad y contacto diario con los miembros de su unidad académica, lo que les permite influir en las personas y en la cultura dentro del área de la que son responsables [9], ayudando así a respaldar una relación digna de confianza entre la academia y los recursos humanos [13].

Por último, se encuentran los líderes académicos. A este respecto, Salmi y Pham [4] sostienen que los líderes docentes desempeñan un papel importante en el mantenimiento de los estándares académicos y en el desarrollo de programas de investigación y capacitación, los cuales son considerados como la actividad principal de las instituciones de educación superior.

En definitiva, el liderazgo se considera una habilidad altamente necesaria para mantener la competitividad de las instituciones, ya que el rol del líder no solo se limita a administrar los procesos de implementación, sino que también se encarga de apoyar el desarrollo, gestionar el cambio y construir una cultura de 
calidad [7], lo que permite así generar nuevas ideas y aumentar el esfuerzo para el logro de la efectividad institucional [13]. En este sentido son múltiples los autores que recomiendan el incentivo de esta habilidad tanto en los líderes formales como en los académicos en general $[9,14,15,16,17]$.

\section{METODOLOGÍA}

Se llevó a cabo un análisis bibliométrico de la literatura en inglés y español entre los períodos del 2019 a julio del 2020. Esto con el fin de realizar un análisis de contenido para la identificación de las principales líneas de investigación del liderazgo en las instituciones de educación terciaria en este período.

En este sentido se trabajó con las bases de datos de Web of Science (WoS), Scopus y SciELO, en las cuales se realizaron las búsquedas de los términos "leadership" y "higher education institutions", "leadership" y "universities", y por último "leadership" y "community colleges". Asimismo, se filtraron dichas búsquedas por tipo de documento 'Article', como por la palabra clave 'Leadership' para Scopus, y categoría de 'Education - Educational Research' para WoS y SciELO.

Para el análisis e interpretación de datos se utilizó el paquete Bibliometrix R de RStudio, el cual es una herramienta de código abierto orientado a la investigación cuantitativa en cienciometría y bibliometría, y que permite describir y realizar un seguimiento de las publicaciones para detectar tendencias en el campo de estudio.
En la Tabla 1 se presentan las dimensiones consideradas para el análisis.

\section{RESULTADOS}

Fruto de la búsqueda llevada a cabo en la base de datos WoS bajo los términos de "leadership" y "higher education institutions", se encontraron 142 artículos entre los años 2010 y 2020, de los cuales aproximadamente el 37,3\% de ellos (53 artículos) se publicaron entre el período 2019-julio 2020. Ahora bien, al realizar la búsqueda bajo los conceptos de "leadership" y "universities", la base de datos arroja la presencia de 559 artículos en los últimos diez años, de los cuales 161 corresponden al período estudiado (lo que equivale aproximadamente al 28,8\% del total). Por su parte, al realizar la búsqueda del término "leadership" y "community colleges" bajo las mismas condiciones, la base de datos arroja la presencia de 32 artículos durante la última década, de los cuales el 25\% corresponden al período 2019 y julio del 2020 (8 artículos).

Por otro lado, al utilizar la base de datos Scopus, la búsqueda de los conceptos "leadership" y "higher education institutions" arroja un total de 121 artículos en los últimos 10 años, de los cuales 32 de ellos (aproximadamente el 26,4\%) corresponden al período 2019-julio 2020. A su turno, al realizar la búsqueda de "leadership" y "universities", se obtienen 3.608 artículos durante la última década, de los cuales 839 corresponden al período estudiado (lo que equivale aproximadamente al 23,3\% del total). De igual manera, si se emplea la búsqueda bajo los conceptos de "leadership" y "community

Tabla 1. Dimensiones y variables consideradas en la revisión.

\begin{tabular}{|l|l|l|}
\hline \multicolumn{1}{|c|}{ Dimensión } & \multicolumn{1}{|c|}{ Descripción } & \multicolumn{1}{c|}{ Variables de interés } \\
\hline Quién y cuándo se investiga. & $\begin{array}{l}\text { Dimensión que da cuenta de aspectos } \\
\text { básicos de las publicaciones. }\end{array}$ & $\begin{array}{l}\text { Año de publicación. } \\
\text { Autores involucrados. } \\
\text { Afiliaciones de los autores. }\end{array}$ \\
\hline Qué y dónde se investiga. & $\begin{array}{l}\text { Dimensión que aborda el objetivo } \\
\text { de las publicaciones, así como el } \\
\text { contexto en que estas se enfocan. }\end{array}$ & $\begin{array}{l}\text { Perspectiva desde el que se aborda liderazgo. } \\
\text { Contexto en el que se realiza la investigación } \\
\text { (país). }\end{array}$ \\
\hline Dónde se publica. & $\begin{array}{l}\text { Dimensión referida a las revistas } \\
\text { nacionales e internacionales donde } \\
\text { se publican los artículos sobre la la } \\
\text { temática estudiada. }\end{array}$ & Nombre de revista. \\
Factor de impacto.
\end{tabular}

Fuente: Basado en Weinstein et al. ([18]). 
colleges", la base de datos arroja la presencia de 25 artículos durante los últimos 10 años, de los cuales el $8 \%$ corresponden al período 2019-julio 2020, es decir, 2 artículos.

Finalmente, la búsqueda de "leadership" y "higher education institutions" en la base de datos SciELO, indica la existencia de 14 estudios entre los años 2010-2020, de los cuales aproximadamente el $28,6 \%$ de ellos (4 artículos) se publicaron en el período estudiado. Por otro lado, si se realiza la búsqueda de "leadership" y "universities" bajo las mismas condiciones, arroja la presencia de 23 artículos en la última década, de los cuales 5 de ellos pertenecen al período 2019-julio 2020 (lo que equivale aproximadamente al 21,7\% del total). Por último, si la búsqueda se realiza bajo los conceptos de "leadership" y "community colleges", SciELO no arroja ningún artículo en los últimos años.

En la Figura 1 se presentan los resultados obtenidos según base de datos analizadas y tipo de búsqueda, representadas como, I: "leadership" y "higher education institutions", II: "leadership" y "universities" y III: "leadership" y "community colleges".

En el caso de WoS, las diez revistas que más están publicando artículos acerca del liderazgo en el ámbito de las universidades se presentan en la Figura 2. En relación a la búsqueda en los "community colleges" se destaca la revista "Community College Journal of Research and Practice", ya que es aquí donde se publican la gran mayoría de los artículos de este tema en el período estudiado ( 5 de 8 publicaciones).

En la Figura 3 se presentan los factores de impacto de las revistas antes mencionadas.

En relación al liderazgo en universidades, la Figura 4 muestra los países con mayor producción científica en este tema (según autores de las publicaciones).

Por otro lado, la Tabla 2 indica las principales afiliaciones de los autores. Se hace una distinción dependiendo del tipo de búsqueda (I: "leadership" y "higher education institutions", II: "leadership" y "universities", III: "leadership" y "community colleges").

Por último, la Figura 5 muestra la nube de palabras según la búsqueda de liderazgo en universidades en la base de datos WoS.

Para el caso de Scopus, las diez revistas que más están publicando artículos acerca del liderazgo en las universidades se presentan en la Figura 6. Aquí se puede destacar que el estudio del liderazgo en el contexto médico ha tomado gran relevancia en el período estudiado.

En la Figura 7 se presentan los factores de impacto de las revistas antes mencionadas.

En relación al liderazgo en universidades, la Figura 8 muestra los países con mayor producción científica en este tema (según autores de los artículos).

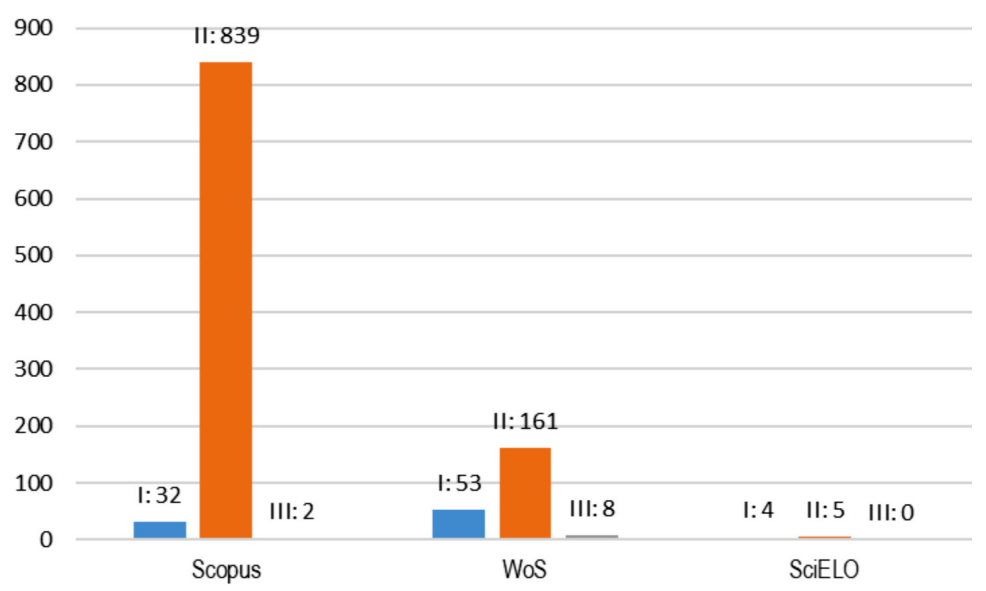

Figura 1. Número de artículos período 2019-julio 2020 según tipo de búsqueda. 
Fuente: Basado en Bibliometrix.

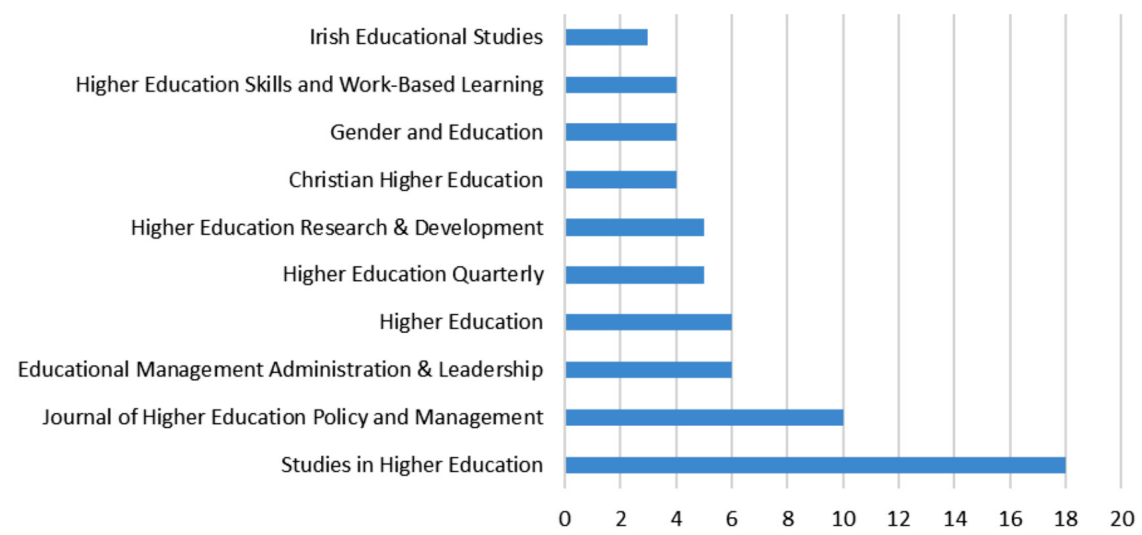

Figura 2. Fuentes más relevantes período 2019-julio 2020 en base de datos WoS.

Fuente: Basado en Bibliometrix.

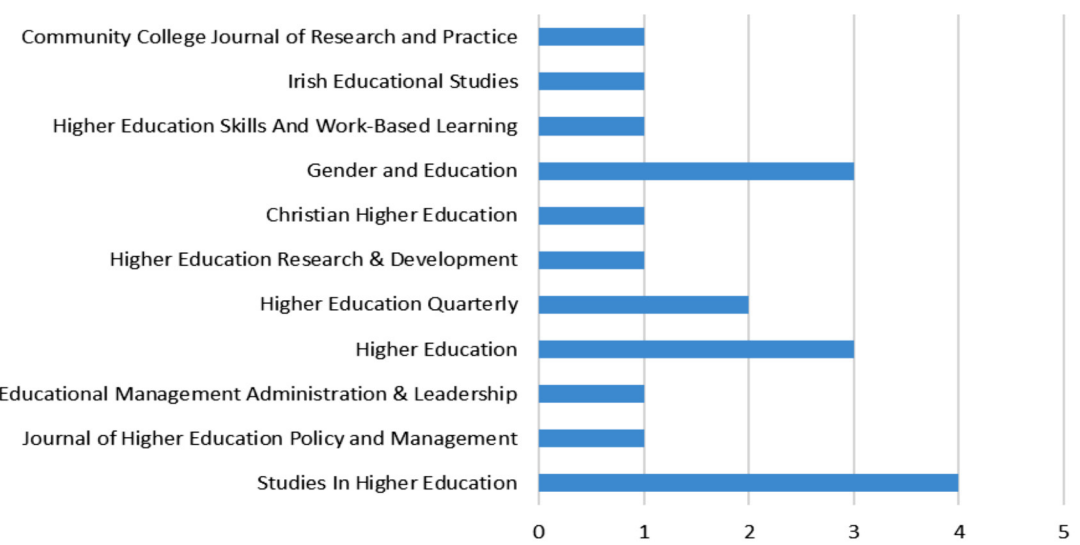

Figura 3. Factor de impacto (h-index) de revistas en WoS.

Fuente: Basado en Bibliometrix.

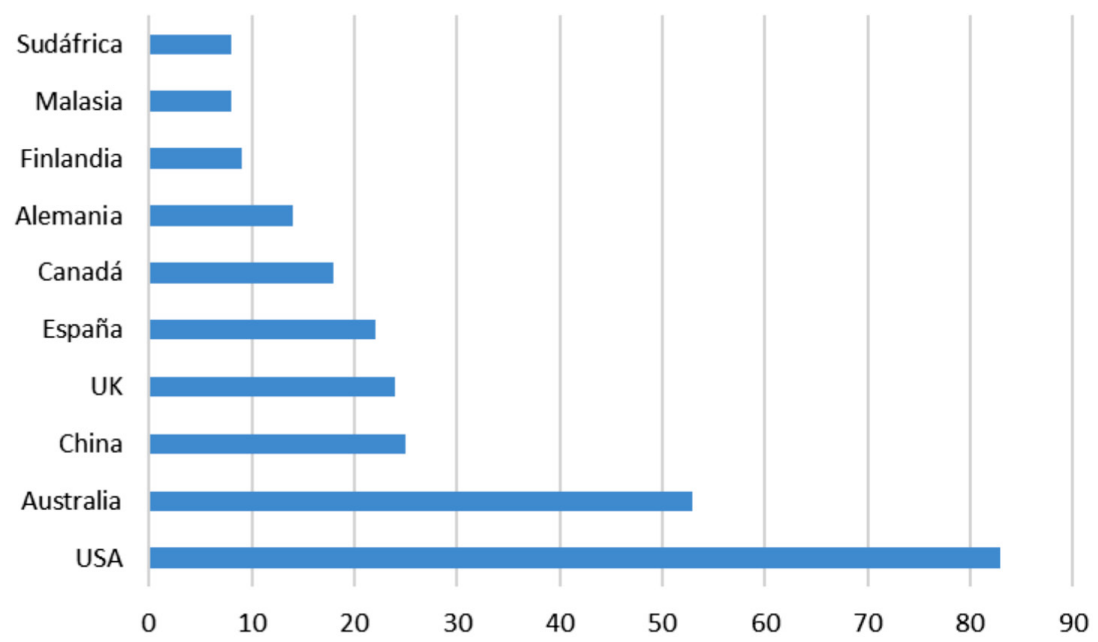

Figura 4. Producción científica por países según autores (WoS). 
Tabla 2. Afiliaciones más relevantes por tipo de búsqueda (WoS).

\begin{tabular}{|l|l|l|}
\hline Tipo de Búsqueda & \multicolumn{1}{|c|}{ Afiliaciones } & \multicolumn{1}{c|}{ País } \\
\hline \multirow{4}{*}{ I } & Kristianstad University & Suecia \\
\cline { 2 - 3 } & Hawassa University & Etiopía \\
\cline { 2 - 3 } & Lund University & Suecia \\
\cline { 2 - 3 } & Sam Houston State University & Estados Unidos \\
\cline { 2 - 3 } & University of South Australia & Australia \\
\hline \multirow{4}{*}{ II } & La Trobe University & Australia \\
\cline { 2 - 3 } & University of Toronto & Canadá \\
\cline { 2 - 3 } & Leuphana University of Lüneburg & Alemania \\
\cline { 2 - 3 } & University of Hong Kong & China \\
\cline { 2 - 3 } & University of Wisconsin & Estados Unidos \\
\hline \multirow{4}{*}{ III } & Florida State University & Estados Unidos \\
\cline { 2 - 3 } & College of William and Mary & Estados Unidos \\
\cline { 2 - 3 } & University of Texas at Arlington & Estados Unidos \\
\cline { 2 - 3 } & Adrian College & Estados Unidos \\
\cline { 2 - 3 } & Florida Atlantic University & Estados Unidos \\
\hline
\end{tabular}

Fuente: Basado en Bibliometrix.

Fuente: Bibliometrix.

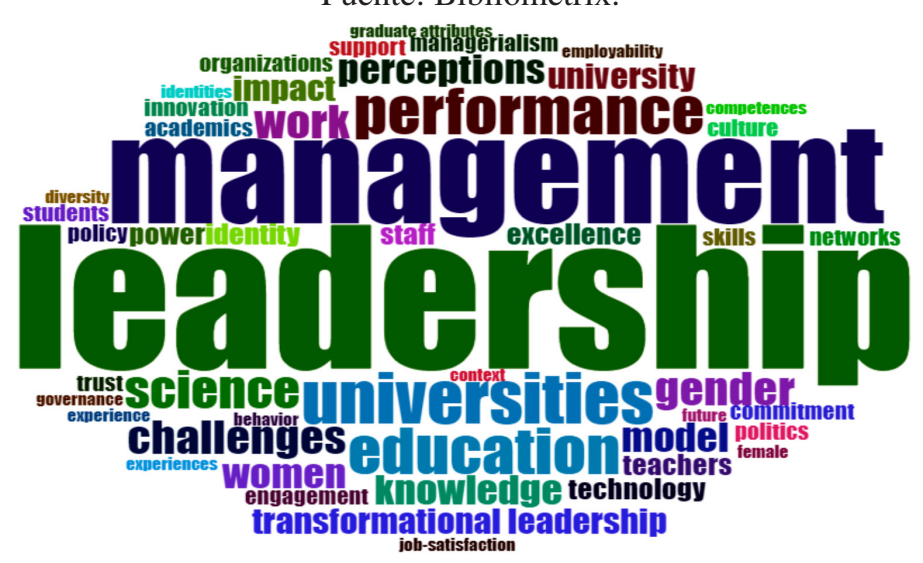

Figura 5. Nube de palabras (WoS).

Por otro lado, la Tabla 3 muestra las principales afiliaciones de los autores. Se hace una distinción dependiendo del tipo de búsqueda (I: "leadership" y "higher education institutions", II: "leadership" y "universities", III: "leadership" y "community colleges").

Por último, la Figura 9 muestra la nube de palabas según la búsqueda de liderazgo en universidades en la base de datos Scopus.

Ahora bien, al realizar el análisis de contenido de los artículos del período 2019-julio 2020 de las bases de datos WoS, Scopus y SciELO, es posible encontrar cuatro líneas de investigación que emergen con fuerza.

Un primer grupo consiste en investigaciones que buscan identificar los efectos (directos o indirectos) que tienen los estilos de liderazgo en distintos aspectos de interés, como lo son en las actitudes positivas de los miembros de la comunidad académica (compromiso, innovación, motivación, satisfacción, creatividad, niveles de estrés, identidad con la organización y desempeño) y en los resultados deseados para las instituciones de educación terciaria 
Fuente: Basado en Bibliometrix.

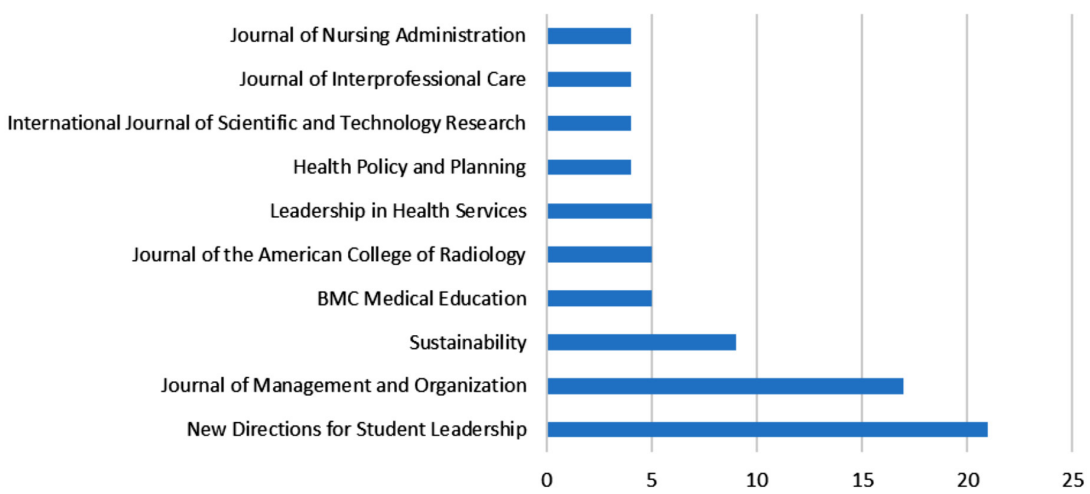

Figura 6. Fuentes más relevantes período 2019-julio 2020 en base de datos Scopus.

Fuente: Basado en Bibliometrix.

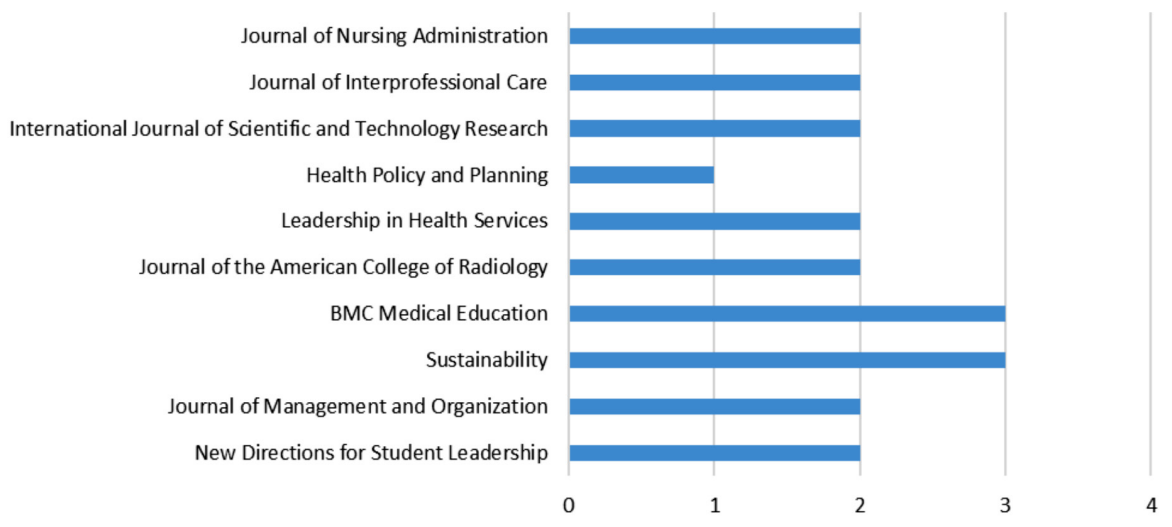

Figura 7. Factor de impacto (h-index) de revistas en Scopus.

Fuente: Basado en Bibliometrix.

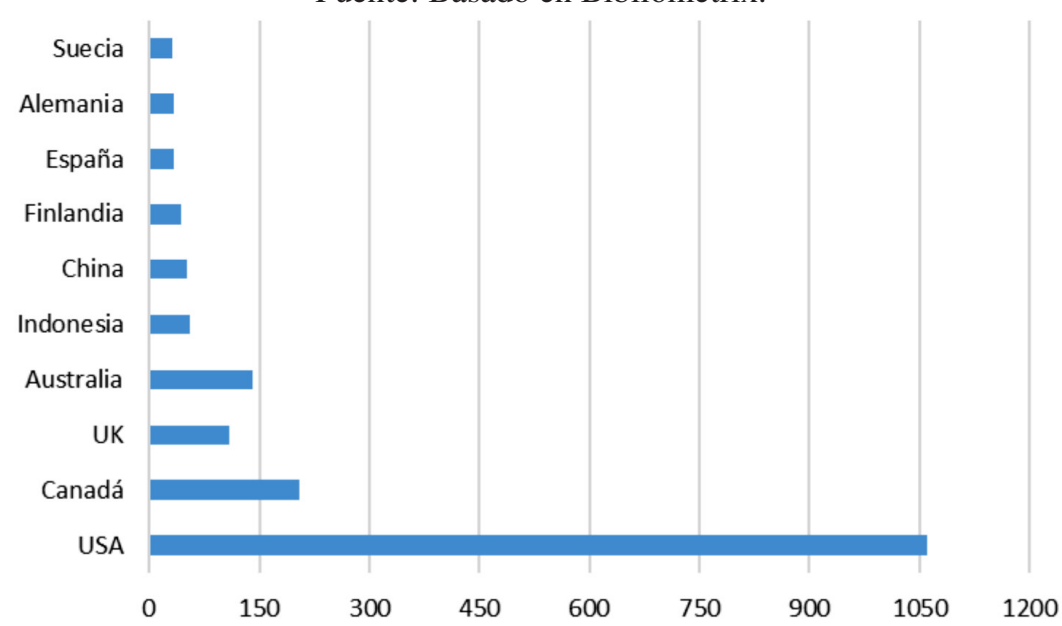

Figura 8. Producción científica por países según autores (Scopus). 
Tabla 3. Afiliaciones más relevantes por tipo de búsqueda (Scopus).

\begin{tabular}{|c|l|l|}
\hline Tipo de Búsqueda & \multicolumn{1}{|c|}{ Afiliaciones } & \multicolumn{1}{c|}{ País } \\
\hline \multirow{4}{*}{ I } & Kennesaw State University & Estados Unidos \\
\cline { 2 - 3 } & University of Hong Kong & China \\
\cline { 2 - 3 } & West Virginia University & Estados Unidos \\
\cline { 2 - 3 } & Saint James School of Medicine & UK \\
\cline { 2 - 3 } & University of Leicester & UK \\
\hline \multirow{4}{*}{ II } & University of Toronto & Canadá \\
\cline { 2 - 3 } & University of California & Estados Unidos \\
\cline { 2 - 3 } & University of Michigan & Estados Unidos \\
\cline { 2 - 3 } & Harvard Medical School & Estados Unidos \\
\cline { 2 - 3 } & Massachusetts General Hospital & Estados Unidos \\
\hline \multirow{4}{*}{ III } & American University & Estados Unidos \\
\cline { 2 - 3 } & College of William and Mary & Estados Unidos \\
\hline
\end{tabular}

Fuente: Basado en Bibliometrix.

Fuente: Bibliometrix.

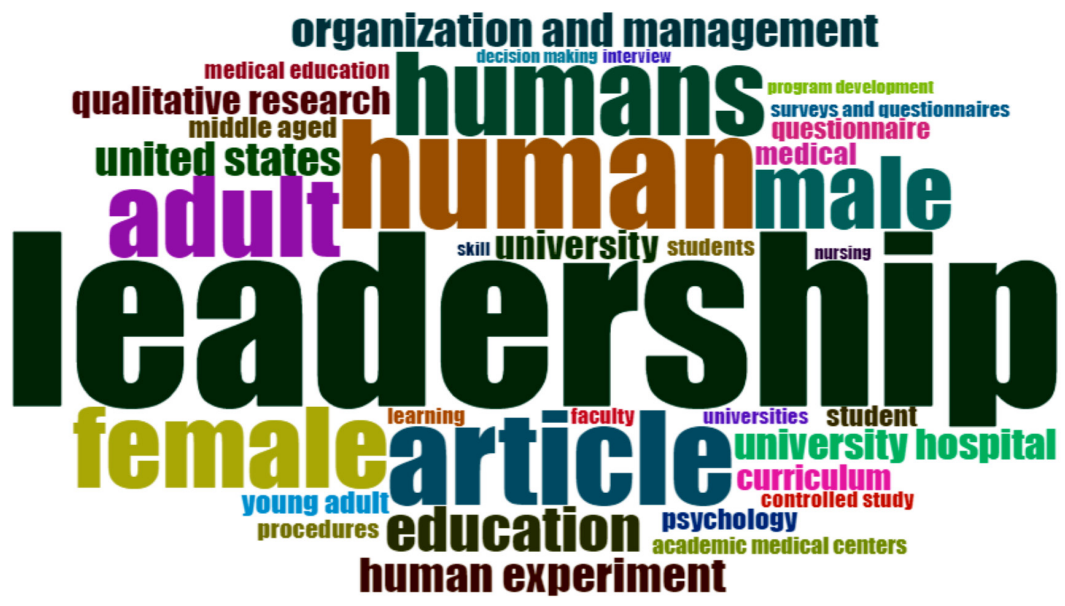

Figura 9. Nube de palabras (Scopus).

(producción científica, responsabilidad social corporativa, logro de la calidad, flexibilidad, gestión y efectividad institucional). En este contexto, se reconocen investigaciones en países como Estados Unidos y Qatar [19, 20], China [12, 21, 22], Suecia [23], Malasia [24], Indonesia [25], Filipinas [26], Vietnam [27, 28], India [29], Palestina [30], Irán [31, 32], Irak [33, 34, 35], Pakistán [13, 36, 37, 38, 39], Jordania [40], Nigeria [41], España [42, 43], México [44], Colombia [45] y Chile [46].

Una segunda línea de investigación aborda los programas de aprendizaje y desarrollo implementados en las instituciones de educación terciaria para incentivar el liderazgo en los estudiantes, así como en las iniciativas para fomentar el desarrollo de esta habilidad en los docentes. Aquí se encuentran estudios realizados en países como Estados Unidos [47, 48, 49, 50, 51, 52], Canadá [53], Australia [9, 54], Nueva Zelanda [16], Finlandia [55], Reino Unido [56], Suecia [57], Ucrania [58], China [59], Malasia [60], Sudáfrica [14], Emiratos Árabes [61] y España $[62,63]$. Igualmente, se encuentran iniciativas que cuentan con participación internacional (ejemplo [64]).

La tercera línea de investigación que se identifica consiste en el liderazgo de las mujeres en la academia. Principalmente, se estudia el tema desde 
una perspectiva crítica, haciendo énfasis en las dificultades que enfrentan las mujeres (como los estereotipos de género e impedimentos de naturaleza cultural) para acceder a los rangos directivos, lo cual hace que estas tengan una subrepresentación en los puestos de liderazgo de las instituciones de educación terciaria. En este contexto, se reconocen investigaciones en países como Estados Unidos [65, 66, 67, 68], Canadá [69], Australia y Nueva Zelanda [70], Reino Unido e Italia [71], Irlanda [72, 73], China [74, 75], Indonesia [76], en países de Asia Central como Kazajistán y Kirguistán [77], Arabia Saudita [78], India [79], Sudáfrica [17, 80] y México [81, 82], entre otros.

Finalmente, se identifica una línea de investigación centrada en las actitudes, competencias, conocimientos y características que deben presentar los líderes para ser efectivos en el ámbito académico. Aquí los estudios empíricos tienen distintos focos, ya sea en centrarse en los rasgos propios de la persona que se enfrenta al cargo de líder como en las actitudes que estos tienen hacia los académicos, estudiantes, miembros de un equipo de trabajo o institución en general. Bajo esta perspectiva, se pueden nombrar investigaciones realizadas en países como Estados Unidos [83, 84, 85], Reino Unido [86], Australia [87], Canadá [88], China [89], India [90, 91], Indonesia [92, 93], Nigeria [2], España [94], Cuba [95] y México [96]. De igual manera se han realizado estudios con cooperación internacional (ejemplo [97]).

\section{CONCLUSIONES}

El análisis realizado refleja que el liderazgo ha sido estudiado en múltiples contextos culturales, por lo que se confirma que es una temática que posee gran relevancia global. Asimismo, los resultados indican que la mayoría de los artículos de investigación científica sobre el liderazgo se centran en las universidades, relegando a un nivel secundario a los otros tipos de instituciones de educación terciaria.

Consecuentemente, al revisar en detalle los estudios de las bases de datos Web of Science (WoS), Scopus y SciELO fue posible identificar cuatro líneas de investigación que emergen con fuerza en el período 2019-julio 2020, las cuales son: 1) los efectos (directos o indirectos) que tienen los estilos de liderazgo en distintos aspectos de interés, 2) los programas de aprendizaje y desarrollo implementados en las instituciones de educación terciaria para incentivar el liderazgo en los estudiantes y docentes, 3) el liderazgo de las mujeres en la academia, y 4) las actitudes, competencias, conocimientos y características que deben presentar los líderes para ser efectivos en el ámbito académico.

Una limitación que presenta la investigación es el período acotado con el que se trabaja. De igual manera, la ampliación del rango de estudio, así como el uso de otros métodos de revisión (libros, disertaciones, etc.) y de otras bases de datos, podría arrojar resultados ligeramente diferentes. Es por esto, que los resultados entregados pueden interpretarse como puntos de referencia, los cuales se pueden revisar en profundidad utilizando métodos alternativos de análisis en estudios posteriores.

\section{AGRADECIMIENTOS}

Los autores agradecen a la ANID por el financiamiento del Proyecto FONDECYT Regular No 1180484. A su vez se agradece a ANID PIA CIE 160007 de CONICYT.

\section{REFERENCIAS}

[1] A. Bernasconi y E. Rodríguez-Ponce. "Los desafíos para el gobierno corporativo universitario en la era del conocimiento". Ingeniare: Revista Chilena de Ingeniería. Vol. $26 \mathrm{~N}^{\circ}$ 2, pp. 189-191. 2018.

[2] A.S. Adewale and S. Ghavifekr. "Leadership self-efficacy and staff organizational citizenship behavior in higher education institutions: Experience from Nigeria". International Journal of Leadership in Education. Vol. $22 \mathrm{~N}^{\circ}$ 1, pp. 116-133. 2019.

[3] L. Pedraja-Rejas, Í. Marchioni-Choque, C. Espinoza-Marchant y C. Muñoz-Fritis. "Liderazgo y cultura organizacional como factores de influencia en la calidad universitaria: Un análisis conceptual". Formación Universitaria. Vol. 13 № 5, pp. 3-14. 2020.

[4] J. Salmi and L.T. Pham. "Academic governance and leadership in Vietnam: Trends and challenges" Journal of International and Comparative Education (JICE). Vol. $8 \mathrm{~N}^{\circ} 2$, pp. 103-118. 2019. 
[5] C. Sugrue, T.D. Solbrekke, A. Bergh, M. Sutphen and T. Fossland. "University leaders' talk about institutional missions and academic developers' contributions" European Educational Research Journal. Vol. 18 N $^{\circ}$ 6, pp. 743-759. 2019.

[6] L. Pedraja-Rejas, C. Araneda-Guirriman, A. Bernasconi y P. Viancos. "Liderazgo, cultura académica y calidad de las universidades: Aproximación conceptual y relaciones". Revista Venezolana de Gerencia. Vol. 23, No 1, pp. 184-199. 2018.

[7] K. Alzafari and J. Kratzer. "Challenges of implementing quality in european higher education: An expert perspective". Quality in Higher Education. Vol. $25 \mathrm{~N}^{\circ} 3$, pp. 261288. 2019.

[8] A. Parvin. "Leadership and management in quality assurance: Insights from the context of Khulna university, Bangladesh". Higher education. Vol. 77, pp. 39-756. 2018.

[9] J. Butler. "Learning to lead: A Discussion of development programs for academic leadership capability in australian universities". Journal of Higher Education Policy and Management. Vol. $42 \mathrm{~N}^{\circ} 4$, pp. 424-437. 2019.

[10] S.M. Tellmann, T. Røsdal and N. Frølich. "Professional educational programmes under pressure. Organizational challenges related to strengthening research". Studies in Higher Education. pp. 1-11. 2020.

[11] Y.S. Tsai, O. Poquet, D. Gašević, S. Dawson and A. Pardo. "Complexity leadership in Learning analytics: Drivers, challenges and opportunities". British Journal of Educational Technology. Vol. 50 No 6, pp. 2839-2854. 2019.

[12] Y.-T Huang, H. Liu and L. Huang. "How transformational and contingent reward leaderships influence university faculty's organizational commitment: The mediating effect of psychological empowerment". Studies in Higher Education, pp. 1-18. 2020.

[13] S. Munir, M.A. Yasin, M.S. Shabbir, S.R. Ali, B. Tariq, M.I. Chani, M. Orangzab and M. Abbas. "Mediating role of organizational citizenship behavior on authentic leadership and employee job performance: A study of higher educational institutes in Pakistan". Dilemas Contemporáneos: Educación, Política y Valores. Vol. 6, pp. 1-21. 2019.
[14] F. Cronje and E.M. Bitzer. "Continuous professional learning in private higher education: Making a case for distributed leadership". South African Journal of Higher Education. Vol. $33 \mathrm{~N}^{\circ}$ 2, pp. 52-68. 2019.

[15] D. Fam, E. Clarke, R. Freeth, P. Derwort, K. Klaniecki, L. Kater-Wettstädt, S. JuarezBourke, S. Hilser, D. Peukert, E. Meyer and A.I. Horcea-Milcu. "Interdisciplinary and transdisciplinary research and practice: Balancing expectations of the 'old' academy with the future model of universities as 'problem solvers'". Higher Education Quarterly. Vol. 74 No 1, pp. 19-34. 2019.

[16] K. Thornton. "Thank goodness it isn't just me building a learning community for middle leaders". Journal of Higher Education Policy and Management. Vol. $42 \mathrm{~N}^{\circ} 3$, pp. 316-331. 2020.

[17] N. Toni and A. L. Moodly. "Do institutional cultures serve as impediments for women's advancement towards leadership in south african higher education?". South African Journal of Higher Education. Vol. $33 \mathrm{~N}^{\circ} 3$, pp. 176-191. 2019.

[18] J. Weinstein, G. Muñoz, M. Sembler y J. Marfán. "Una década de investigación empírica sobre el liderazgo educativo en Chile. Una Revisión Sistemática de los Artículos Publicados en Revistas Indexadas (2008-2019)". Calidad en la Educación. $\mathrm{N}^{\mathrm{o}}$ 51, pp. 15-52. 2019.

[19] R.S. Al-Mansoori and M. Koç. "Toward knowledge-based economy: Innovation and transformational leadership in public universities in Texas and Qatar". Sustainability. Vol. 11 NN$^{\circ} 23$, pp. 6721. 2019.

[20] B.S. Sipe. "Embedding design thinking in a culture of innovation at two community colleges". Community College Journal of Research and Practice. Vol. 44 N $^{\circ}$ 9, pp. 657-676. 2019.

[21] J. Zeng and G. Xu. "Ethical leadership and young university teachers' work engagement: A moderated mediation model". International Journal of Environmental Research and Public Health. Vol. $17 \mathrm{~N}^{\circ}$ 1, pp. 1-15. 2020a.

[22] J. Zeng and G. Xu. "How servant leadership motivates innovative behavior: A moderated mediation model". International Journal of Environmental Research and Public Health. Vol. $17 \mathrm{~N}^{\mathrm{o}}$ 13, pp. 1-14. $2020 \mathrm{~b}$. 
[23] I. Jensen, C. Bjorklund, J. Hagberg, E. Aboagye and L. Bodin. "An Overlooked Key To Excellence In Research: A longitudinal cohort study on the association between the psycho-social work environment and research performance". Studies in Higher Education. pp. 1-19. 2020.

[24] S. Kaur, J. Kaur, H.J. Singh, J. Singh, K. Singh and S. Kumar, S. "Leadership styles and educators commitment in private universities in Malaysia". TEST Engineering \& Management. Vol. $82 \mathrm{~N}^{\circ} 1-2$, pp. 917-930. 2020.

[25] Nasrun, D.F.P. Ambarita, N.B. Nasution and H.P. Tambunan. "The effect of dean leadership, on the performance of lecturers working in the faculty of education in Medan state university". International Journal of Innovation, Creativity and Change. Vol. 5 $\mathrm{N}^{\circ}$ 5, pp. 515-530. 2019.

[26] M.C. Celdran. "Leadership styles and job satisfaction in the colleges of nursing among the universities in Zamboanga city". International Journal of Innovation, Creativity and Change. Vol. $11 \mathrm{~N}^{\circ}$ 7, pp. 444-455. 2020.

[27] H.T.T. Suong, D.D. Thanh and T.T.X. Dao. "The impact of leadership styles on the engagement of cadres, lecturers and staff at public universities-evidence from Vietnam". Journal of Asian Finance, Economics and Business. Vol. $6 \mathrm{~N}^{\mathrm{o}}$ 1, pp. 273-280. 2019.

[28] T.H. Vu, M.T. Vu and V.N. Hoang. "The Impact of transformational leadership on promoting academic research in higher educational system in vietnam". Management Science Letters. Vol. $10 \mathrm{~N}^{\mathrm{o}}$ 3, pp. 585-592. 2020.

[29] N. Garg and S. Gera. "Gratitude and leadership in higher education institutions". Journal of Applied Research in Higher Education, pp. 1-12. 2019.

[30] M. Aboramadan, K. Dahleez and M. Hamad. 'Servant leadership and academics' engagement in higher education: Mediation analysis". Journal of Higher Education Policy and Management, pp. 1-17. 2020.

[31] A.P. Hesar, M.M. Seyed-Abbaszadeh, A. Ghalei and H. Ghalavandi. "Investigating the relationship between transformational leadership style and organizational identity of faculty members in the state universities of west Azerbaijan province, Iran". Serbian Journal of Management. Vol. $14 \mathrm{~N}^{\circ}$ 1, pp. 157176. 2019.

[32] M. Ranjbar, S. Rafiei, M. Shafiei and V. Kargar. "Transformational leadership style and employee creativity: A case study in Yazd medical university". The Health Care Manager. Vol. $38 \mathrm{~N}^{\circ}$ 3, pp. 282-288. 2019.

[33] A.S. Jameel and A.R. Ahmad. "The mediating role of job satisfaction between leadership style and performance of academic staff'. International Journal of Psychosocial Rehabilitation. Vol. 24No 4, pp. 2399-2414. 2020.

[34] M.A. Waham, R.A. Rahman and W.S.W. Mustaffa. "The effect of transformational leadership on organizational performance through the mediating role of organizational culture in higher education institutions in Iraq". International Journal of Psychosocial Rehabilitation. Vol. 24 N$^{\circ}$ 8, pp. 13638-13651. 2020.

[35] O.H. Waisy and C.C. Wei. "The Moderating Role of Type of University in the relationship between transformational leadership and supply chain management: Evidence from universities in the Kurdistan region of Iraq". International Journal of Supply Chain Management. Vol. $8 \mathrm{~N}^{\circ}$ 4, pp. 761-771. 2019.

[36] A. Adil and A. Kamal. "Authentic leadership and psychological capital in job demandsresources model among pakistani university teachers". International Journal of Leadership in Education, pp. 1-21. 2019.

[37] M. Farrukh, J.W.C. Lee and I.A. Shahzad. "Intrapreneurial behavior in higher education institutes of Pakistan". Journal of Applied Research in Higher Education. Vol. $11 \mathrm{~N}^{\mathrm{o}} 2$, pp. 273-294. 2019.

[38] S.I.U. Hassan and B.H. Din. "The mediating effect of knowledge sharing among intrinsic motivation, high-performance work system and authentic leadership on university faculty members' creativity". Management Science Letters. Vol. $9 \mathrm{~N}^{\circ}$ 6, pp. 887-898. 2019.

[39] A. Wahab and I. Tyasari. "Entrepreneurial leadership for university leaders: A futuristic approach for pakistani HEIs". Asia Pacific Management Review. Vol. 25 No 1, pp. 54-63. 2020.

[40] K. Alshihabat, and T. Atan. "The mediating effect of organizational citizenship behavior 
in the relationship between transformational leadership and corporate social responsibility practices: Middle eastern example/Jordan". Sustainability. Vol. $12 \mathrm{~N}^{\circ}$ 10, pp. 1-17. 2020.

[41] D.J. Kayode, N.M. Yusoff and A. Veloo. "The mediating role of a quality academic process on the relationship between distributed leadership and the effectiveness of public universities in Nigeria". Kasetsart Journal of Social Sciences. Vol. $40 \mathrm{~N}^{\circ}$ 2, pp. 319-325. 2019.

[42] J. Ballesteros-Rodríguez, N. Díaz-Díaz, I. Aguiar-Díaz and P. De Saá-Pérez. "The role of leadership in the management of conflict and knowledge sharing in the research groups of a Spanish public university". Public Organization Review. Vol. $20 \mathrm{~N}^{\mathrm{o}} 2$, pp. 421-436. 2019.

[43] J. Ballesteros-Rodríguez, P. De Saá-Pérez, N. García-Carbonell, F. Martín-Alcázar and G. Sánchez-Gardey. "The influence of team members' motivation and leaders' behaviour on scientific knowledge sharing in universities". International Review of Administrative Sciences. pp. 1-17. 2020.

[44] C. Saldaña-Orozco, R. Bustos-Saldaña, A. Barajas-Martínez y G. Ibarra-Rentería. "Liderazgo y riesgo psicosocial en instituciones de educación superior en México". Revista Venezolana de Gerencia. Vol. 24, No 88, pp. 1239-1248. 2019.

[45] G. Pérez-Ortega and Z. Moreno-Freites. "Model of relationship of transformational leadership and university management". Dyna. Vol. 86 N$^{\circ}$ 210, pp. 9-16. 2019.

[46] L. Pedraja-Rejas, A. Bernasconi, G. Coluccio-Piñones, Í. Marchioni-Choque, C. Espinoza-Marchant y C. Muñoz-Fritis. "Cultura y estilos de liderazgo en unidades académicas: un estudio en una institución de educación superior". Utopía y Praxis Latinoamericana. Vol. 24, No 4, pp. 25-35. 2019.

[47] M.I. Honig and A. Honsa. "Systems-focused equity leadership learning: Shifting practice through practice". Journal of Research on Leadership Education. Vol. $15 \mathrm{~N}^{\circ} 3$, pp. 192209. 2020.

[48] K. Kearns. "The leadership portfolio program at the university of pittsburgh: Teaching leadership to graduate students". Teaching
Public Administration. Vol. $37 \mathrm{~N}^{\mathrm{o}} 3$, pp. 255273. 2019.

[49] S.I. Nolan-Arañez. "The intersectionality of leadership and spiritual development through mentoring". New Directions for Student Leadership. Vol. $2020 \mathrm{~N}^{\circ}$ 166, pp. 97-110. 2020.

[50] G. Szteinberg, M.D. Repice, C. Hendrick, S. Meyerink and R.F. Frey. "Peer leader reflections on promoting discussion in peer group-learning sessions: Reflective and practiced advice through collaborative annual peer-advice books". CBE-Life Sciences Education. Vol. 19 No 1, pp. 1-13. 2020.

[51] J.Y.Tsoh, A.K. Kuo, J.W. Barr, L. Whitcanack, I. Merry, B.K. Alldredge and A.N. Azzam. "Developing faculty leadership from "within": A 12-Year reflection from an internal faculty leadership development program of an academic health sciences center". Medical Education Online. Vol. $24 \mathrm{~N}^{\mathrm{o}}$ 1, pp. 1-15. 2019.

[52] R.L. Vidra, D.R. Gallagher and V. Wilson. "Acknowledging the challenges of pedagogical innovation: The case of integrating research, teaching, and the practice of environmental leadership". Journal of Environmental Studies and Sciences. Vol. $9 \mathrm{~N}^{\circ} 3$, pp. 270-275. 2019.

[53] A.M.Corriveau. "Developing authentic leadership as a starting point to responsible management: A Canadian University Case Study". The International Journal of Management Education. Vol. $18 \mathrm{~N}^{\mathrm{o}}$ 1, pp. 1-10. 2020.

[54] R. Al-Mahmood, G. Papalia, S. Barry, M. Nguyet Nguyen, J. Roemhild, T. MeehanAndrews, T. Meehan-Andrews, B. Julien, C. Holt, L. Bester, C. Bruce, R. Miles, C. Neilson and J. Louie. "Love acts and revolutionary praxis: Challenging the neoliberal university through a teaching scholars development program". Higher Education Research \& Development. Vol. $39 \mathrm{~N}^{\mathrm{o}}$ 1, pp. 81-98. 2020.

[55] L.-M. Hero and E. Lindfors. "Students' learning experience in a multidisciplinary innovation project". Education + Training. Vol. $61 \mathrm{~N}^{\circ}$ 4, pp. 500-522. 2019.

[56] J. Brant, M. Lamb, E. Burdett and E. Brooks. "Cultivating virtue in postgraduates: An empirical study of the Oxford global leadership initiative". Journal of Moral Education. Vol. $49 \mathrm{~N}^{\circ}$ 4, pp. 415-435. 2019. 
[57] C. Mcgrath, T. Roxå and K. Bolander Laksov. "Change in a culture of collegiality and consensus-seeking: A double-edged sword". Higher Education Research \& Development. Vol. $38 \mathrm{~N}^{\circ}$ 5, pp. 1001-1014. 2019.

[58] O. Soroka, S. Kalaur and A. Balendr. "Diagnostics of leadership qualities of specialists of 'man-man' type of professions in military and civil higher education institutions: Psychological and pedagogical approach". Romanian Journal for Multidimensional Education. Vol. 11, pp. 264-277. 2019.

[59] L. Lin and D.T. Shek. "Does service leadership education contribute to student well-being? A quasi-experimental study based on Hong Kong university students". Applied Research in Quality of Life. Vol. $14 \mathrm{~N}^{\circ}$ 5, pp. 11471163. 2019.

[60] S. Shafee, S. Ghavifekr and Z. Abdullah. "Leadership role of coaches in improving teachers' instructional practices". Malaysian Online Journal of Educational Management. Vol. 7 No 1, pp. 92-112. 2019.

[61] S. Baroudi and S. Arulraj David. "Nurturing female leadership skills through peer mentoring role: A study among undergraduate students in the united Arab Emirates". Higher Education Quarterly. pp. 1-17. 2020.

[62] S. Deer and N. Simpson. "Solvitur ambulando' (it is solved by walking): Learning business leadership on the camino de Santiago". Journal of Management Education. Vol. 44 $\mathrm{N}^{\circ}$ 4, pp. 1-29. 2020.

[63] M. Segovia-Pérez, P. Laguna-Sánchez, and C. De La Fuente-Cabrero. "Education for sustainable leadership: Fostering women's empowerment at the university level". Sustainability. Vol. $11 \mathrm{~N}^{\mathrm{o}}$ 20, pp. 1-14. 2019.

[64] C. Stone-Johnson and S. Hayes. "Using improvement science to (re) design leadership preparation: exploring curriculum change across five university programs". Journal of Research on Leadership Education. pp. 1-21. 2020.

[65] M.Y. Delgado and T. Ozuna Allen. "Case studies of women of color leading community colleges in Texas: Navigating the leadership pipeline through mentoring and culture". Community College Journal of Research and Practice. Vol. $43 \mathrm{~N}^{\circ} 10-11$, pp. 718-729. 2019.
[66] K.A. Longman, A. Drennan, J. Beam and A.F. Marble. "The secret sauce: How developmental relationships shape the leadership journeys of women leaders in christian higher education". Christian Higher Education. Vol. 18 No 1-2, pp. 54-77. 2019.

[67] B.M. Rauhaus and I.A.S. Carr. "The invisible challenges: Gender differences among public administration faculty". Journal of Public Affairs Education. Vol. $26 \mathrm{~N}^{\mathrm{o}}$ 1, pp. 31-50. 2019.

[68] C.M. Sánchez and K. Lehnert. "The unbearable heaviness of leadership: The effects of competency, negatives, and experience on women's aspirations to leadership". Journal of Business Research. Vol. 95, pp. 182-194. 2019.

[69] J. O'dea. "Hazardous Manoeuvres: Thoughts on being a Female University Dean". Irish Educational Studies. Vol. $39 \mathrm{~N}^{\circ}$ 2, pp. 205 219. 2019.

[70] T. Fitzgerald. "Mapping the terrain of leadership: Gender and leadership in higher education". Irish Educational Studies. Vol. 32 $\mathrm{N}^{\circ}$ 9, pp. 221-232. 2020.

[71] E. Spanò. "Femina academica: Women "confessing'leadership in higher education". Gender and Education. Vol. $32 \mathrm{~N}^{\circ}$ 3, pp. 301310. 2020.

[72] J. Harford. "The path to professorship: Reflections from women professors in Ireland". Irish Educational Studies. Vol. 39 $\mathrm{N}^{\circ}$ 2, pp. 193-204. 2020.

[73] P. O'connor. "Creating gendered change in irish higher education: is managerial leadership up to the task?... Irish Educational Studies. Vol. $39 \mathrm{~N}^{\mathrm{o}}$ 2, pp. 139-155. 2019.

[74] S.J. Aiston, C.K. Fo and W.W. Law. "Interrogating strategies and policies to advance women in academic leadership: The case of Hong Kong". Journal of Higher Education Policy and Management. Vol. 42 $\mathrm{N}^{\mathrm{o}}$ 3, pp. 347-364. 2020.

[75] S.J. Aiston and C.K. Fo. "The silence/ing of academic women". Gender and Education. pp. 1-18. 2020.

[76] Z. Sakhiyya and K. Locke. "Empowerment vs. Meritocracy discourses in indonesian public universities: The case of female leaders". Asian Journal of Women's Studies. Vol. 25 $\mathrm{N}^{\circ}$ 2, pp. 198-216. 2019. 
[77] A. Kuzhabekova and A. Almukhambetova. "Women's progression through the leadership pipeline in the universities of Kazakhstan and Kyrgyzstan". Compare: A Journal of Comparative and International Education. pp. 1-19. 2019.

[78] A.H. Gorondutse, H. Hilman, M.G. Salimon, R. Alajmi, F.H. Al Shdaifat and V.V. Kumaran. "Establishing the effect of government support on the relationship between Gender egalitarian and women leadership effectiveness among public universities in KSA". Gender in Management: An International Journal. Vol. $34 \mathrm{~N}^{\mathrm{o}}$ 4, pp. 306-325. 2019.

[79] M. Gandhi and K. Sen. "Missing women in Indian university leadership: Barriers and Facilitators". Educational Management Administration \& Leadership. pp. 1-18. 2020.

[80] R. Bonzet and B.L. Frick. "Gender transformation experiences among women leaders in the western cape TVET sector: A narrative response". Education as Change. Vol. $23 \mathrm{~N}^{\mathrm{o}}$ 1, pp. 1-21. 2019.

[81] L. Castañeda, M. Parga, A. Musalem y C. González. "Mujeres directivas en universidades. Los retos institucionales ante la interseccionalidad del género, las profesiones y las particularidades regionales". RIDE. Revista Iberoamericana para la Investigación y el Desarrollo Educativo. Vol. 9, $\mathrm{N}^{\circ} 18$, pp. 377-391. 2019.

[82] R. Gaete, J.Álvarez y M. Ramírez.“Reflexiones y experiencias de profesoras-investigadoras mexicanas sobre el techo de cristal". Calidad en la Educación. № 50, pp. 457-491. 2019.

[83] E. Alward and Y. Phelps. "Impactful leadership traits of virtual leaders in higher education". Online Learning. Vol. $23 \mathrm{~N}^{\mathrm{o}}$ 3, pp. 72-93. 2019.

[84] L. Artis and S. Bartel. "Filling the leadership pipeline:A qualitative study examining leadership development practices and Challenges Facing Community College presidents in Illinois". Community College Journal of Research and Practice. pp. 1-13. 2020.

[85] D. Tarker. "Transformational leadership and the proliferation of community college leadership frameworks: A systematic review of the literature". Community College Journal of Research and Practice. Vol. 43 N N $^{\mathrm{1}} 10-11$, pp. 672-689. 2019.
[86] K. Gravett, I.M. Kinchin and N.E. Winstone. "'More than customers': Conceptions of students as partners held by students, staff, and institutional leaders". Studies in Higher Education. pp. 1-14. 2019.

[87] T. Mason and B. De La Harpe. "The State of play of associate deans, Learning and teaching, in Australian universities, 30 years on". Higher Education Research \& Development. Vol. $39 \mathrm{~N}^{\circ} 3$, pp. 532-545. 2019.

[88] E. Lavigne and C.M. Sá. "The changing roles and qualifications of Canadian university presidents and provosts". Higher Education. pp. 1-14. 2020.

[89] N.B.K. Dinh, A. Caliskan, and C. Zhu. "Academic leadership: Perceptions of academic leaders and staff in diverse contexts". Educational Management Administration \& Leadership, pp. 1-21. 2020.

[90] D.V. Banker, and K.T. Bhal. "Creating world class universities: Roles and responsibilities for academic leaders in India”. Educational Management Administration \& Leadership, pp. 1-21. 2019.

[91] N. Rahnuma. "Evolution of quality culture in an HEI: Critical insights from university staff in Bangladesh". Educational Assessment, Evaluation and Accountability. Vol. 32, pp. 53-81. 2020.

[92] S. Pawenang, S. Supriyati and L. Sobri. "Role of leadership in transformation of higher education in Indonesia". TEST Engineering \& Management. Vol. 83, pp. 1017-1024. 2020.

[93] F.A. Yusuf. "Effects of lecturer trust on leadership at private universities in Banten". International Journal of Scientific and Technology Research. Vol. 9 N$^{\circ} 3$, pp. 14481451. 2020.

[94] I. Martínez-García, M.T. Padilla-Carmona and E. Alastor. "Understanding governance at university: From management to leadership". Journal of Applied Research in Higher Education. Vol. 11 No 3, pp. 466-480. 2019.

[95] E. Moreno-Socorro y R. Urquiza. "Evaluación del liderazgo de los directivos de la educación superior cubana: Un Procedimiento Metodológico". Revista Cubana de Educación Superior. Vol. 39, No 1, pp. 1-18. 2020. 
[96] R. Badillo-Vega and A. Buendía-Espinosa. “The leadership roles of Mexico's University presidents". Studies in Higher Education, pp. 1-16. 2020.

[97] W. Leal Filho, J.H. Pires Eustachio, A.C. Ferreira, M. Will, A. Lange Salvia, I.S.
Rampasso, R. Anholon, J. Platje and M. Kovaleva. "Sustainability leadership in higher education institutions: An overview of challenges". Sustainability. Vol. $12 \mathrm{~N}^{\circ}$ 9, pp. 1-19. 2020. 